\title{
Erratum to: Scalar-vector-tensor gravity from preferred reference frame effects
}

\section{Hossein Ghaffarnejad}

Received: 30 July 2009 / Accepted: 17 September 2009 / Published online: 20 October 2009

(C) Springer Science+Business Media, LLC 2009

\section{Erratum to: Gen Relativ Gravit (2008) 40:2229-2239 DOI 10.1007/s10714-008-0630-8}

The relation (3.3) should be corrected with $\xi=1$. In that case the action functional defined by (3.5) becomes

$$
I_{G J B D}=\frac{3}{8 \pi} \int d x^{4}\left\{\frac{\omega a^{3} \dot{\phi}_{L}^{2}}{6 \phi_{L}}+\phi_{L} a \dot{a}^{2}\right\} .
$$

Corrected forms of the equations defined by (3.6) and (3.7) will be, respectively,

$$
\frac{2 \ddot{a}}{a}+\frac{2 \dot{a}^{2}}{a^{2}}+\frac{2 \dot{a} \dot{\phi}_{L}}{a \phi_{L}}-\frac{\omega \dot{\phi}_{L}^{2}}{2 \phi_{L}^{2}}=0
$$

and

$$
\frac{\omega \ddot{\phi}_{L}}{3 \phi_{L}}-\frac{\omega \dot{\phi}_{L}^{2}}{6 \phi_{L}^{2}}+\frac{\omega \dot{a} \dot{\phi}_{L}}{a \phi_{L}}-\frac{\dot{a}^{2}}{a^{2}}=0 .
$$

The online version of the original article can be found under doi:10.1007/s10714-008-0630-8.

H. Ghaffarnejad ( $\square)$

Physics Department, Semnan University, P. O. Box 35195-363, Semnan, Iran

e-mail: hghafarnejad@yahoo.com 
The equations defined by (3.9) and (3.10) should be changed by the following equations, respectively:

$$
2 \dot{U}+4 U^{2}+2 U V-\frac{\omega}{2} V^{2}=0
$$

and

$$
\frac{\omega}{3} \dot{V}+\frac{\omega}{6} V^{2}+\omega U V-U^{2}=0 .
$$

The relations defined by (3.11) and (3.12) should be replaced by the following forms, respectively:

$$
\Delta=\frac{V}{U}=-12\left(2+\frac{1}{\omega}\right)
$$

and

$$
\omega_{ \pm}=\frac{-275 \pm i \sqrt{407}}{528}
$$

which cannot be take a physical solution. Corrected forms of the relation defined by (3.13) become $\Delta_{ \pm}=\frac{-13 \pm i \sqrt{23}}{4}$ (one can obtain easily a singular contracting vacuum solution as $a=a_{0}\left(t / t_{0}\right)^{x}$ and $\phi^{L}=\phi_{0}^{L}\left(t / t_{0}\right)^{y}$ where dynamical equations leads to $x<0$ and $0<y<1$ or $y>2$. Also the dynamical equations defined by (0.2) and (0.3) leads to a singular expanding vacuum solution with choice of $x>0$ and $1<y<2$ ). Time-time component of the stress tensor defined by (3.15) is equal to the Hamiltonian density of the action functional defined by (3.5) such as follows:

$$
\rho(t)=\frac{3}{8 \pi}\left\{\frac{\omega \dot{\phi}_{L}^{2}}{6 \phi_{L}}+\frac{\phi_{L} \dot{a}^{2}}{a^{2}}\right\}
$$

which should be replaced with (3.17). In the relation (3.19) we will have $\phi^{L}(t)=$ $\phi_{0}^{L}\left(t / t_{0}\right)^{2-3 \lambda}$. Corrected form of the equation (3.20) is obtained as

$$
\lambda=\frac{6 \omega \pm \sqrt{48(3 \omega+2) \pi \rho_{0} t_{0}^{2} / \phi_{0}^{L}-24 \omega}}{3(3 \omega+2)} .
$$

The relation defined by (3.22) should be replaced by the following equation:

$$
\omega>-\frac{2}{3}, \quad \phi_{0}^{L}=\frac{16 \pi \rho_{0}}{3(3 \omega+2) \sigma^{2}} .
$$

The above condition predicts that for $\phi_{0}^{L}>0$ and $\omega<-\frac{2}{3}$, there should be negative mass densities $\rho_{0}<0$, in the universe! But it corresponds to $\phi_{0}^{E}>0$ when we use (3.23) with $\xi=1$. 
The relation (3.24) should be corrected as

$$
\phi_{0}^{E}=-\frac{16 \pi \rho_{0}}{3(3 \omega+2) \sigma^{2}} .
$$

Relations defined by (3.27) and (3.28) should be changed, respectively, such as follows:

$$
\varepsilon_{ \pm}=\frac{4 \omega \pm \sqrt{8 \pi \rho_{0} t_{0}^{2}(8 \omega+3) / \phi_{0}^{L}-6 \omega}}{8 \omega+3}
$$

and

$$
\phi_{0}^{L} \leq 4 \pi \rho_{0} t_{0}^{2}\left(\frac{8}{3}+\frac{1}{\omega}\right) .
$$

The relation defined by (3.30) should be corrected as

$$
\omega>-\frac{3}{8}, \quad \phi_{0}^{L}=\frac{8 \pi \rho_{0}}{(8 \omega+3) \zeta^{2}}
$$

